\title{
Representation of Gravity and the Intrinsic Property of the Gravitational Mass
}

\author{
Haitao Gao \\ Institute of Chemical Industry, Beifang University of Nationalities, Yinchuan, China
}

Email address:

916632807@qq.com

To cite this article:

Haitao Gao. Representation of Gravity and the Intrinsic Property of the Gravitational Mass. American Journal of Astronomy and Astrophysics. Vol. 6, No. 3, 2018, pp. 49-56. doi: 10.11648/j.ajaa.20180603.11

Received: August 28, 2018; Accepted: September 10, 2018; Published: October 10, 2018

\begin{abstract}
The space-time structure of gravity mass is analyzed in this paper. It is proposed that "spin angular momentum and rotational angular momentum" are intrinsic properties of gravitational mass, and "curvature and gravitational effect" are representations of gravitational mass. The theoretical basis of the universal gravitation constant $(\mathrm{G}=1 / 16 \pi \mathrm{C})$ is found by studying the expression of gravity mass, and the curvature equation and time effect equation of gravity space are derived. In comparison with the experimental results of general relativity, The relative error of the calculated result of curvature equation of gravity space with the observation result of gravitational deflection of starlight near the sun are less than $8 \%$, Otheres experiments were quite different. However, the intrinsic correlation of the gravitational redshift experiment of the $\gamma$ radiation on the surface of the earth is consistent with the calculation results of the time curvature equation. The results of this study show that space curvature is the root of gravity. The propagation of gravity is the transfer of space curvature, and there is no "graviton" that transmits the gravitational interaction. Gravitational effects can be observed through supernova explosions and neutron star formation in the universe.
\end{abstract}

Keywords: Gravitation Constant, the Curvature of Space, Angular Momentum, Intrinsic Properties

\section{Preface}

\subsection{Gravity and General Relativity}

Since Newton's law of gravitation and general relativity [1, 2] were proposed, scientists have conducted extensive research and discussion on the phenomenon of gravitation. The observation of gravitational waves [3] confirms the validity of general relativity, However, the "gravitons" [4] predicted by general relativity were not discovered.

In all theories, mass is defined as a fundamental being. Gravity as a property of mass is directly observed and referenced, and no scientist thinks about the source of mass and the nature of gravity.

In the law of universal gravitation and general relativity, the universal gravitation constant is directly introduced as a fundamental constant of physics. Scientists have not discussed the causal principle of the existence of the universal gravitation constant.

According to the "first principles" of scientific discovery, we should further study the sources of mass, gravity and the constant of gravitation, and look for the more basic principles of causality between them.

\subsection{The Basic Principles of the Matter Spatial Theory}

In this paper, the mechanism of gravity is discussed on the basis of the Matter Spatial Theory [5], and the gravitational field equation is established by simple mathematical methods and compared with the gravity verification experiment of general relativity.

In accordance with the basic principles of the Matter Spatial Theory, this paper adopts the following basic conventions:

(1) Space is a physical existence;

(2) "Force" is the action to space, and "force" is equal to the reciprocal value of "time"; in the absence of special description, "force" is the reciprocal of time;

(3) There is a steady velocity field in $3 \mathrm{~d}$ space, whose intensity is the speed of light $C$.

(4) Mass is the spatial property of force applied to space generation. There are one-dimensional to four-dimensional masses in the universe, which correspond to velocities, light, electricity and 
gravitational masses.

(5) The "causality principle" of physical changes.

(6) The "simplicity principle" of physical changes.

(7) The micro world and the macro world follow the same legal system.

\section{The Basic Unit of Gravitational Mass}

The definition of the Matter Spatial Theory: "force" ACTS on four-dimensional space and constructs four-dimensional mass. The mass of gravity is mathematically expressed as "force" times the volume of a four dimensional space, the four dimensional mass is the gravitational mass. According to the derivation of the Matter Spatial Theory, the gravitational mass is expressed as follows:

$$
\mathrm{M}=\frac{4}{3} \pi \cdot \mathrm{C} \cdot \mathrm{a}_{0}{ }^{3} \cdot \mathrm{b}_{0}{ }^{3}=\frac{4}{3} \pi \cdot \mathrm{F} \cdot \mathrm{x} \cdot \mathrm{a}_{0}{ }^{3} \cdot \mathrm{b}_{0}{ }^{3}
$$

In the form: $\mathrm{M} \sim$ gravity quality; $\mathrm{C} \sim$ the speed of light; $\mathrm{a}_{0} \sim$ the radius of the gravity mass unit; $\mathrm{b}_{0} \sim$ density coefficient of gravity mass unit; $\mathrm{F} \sim$ the force acting on the gravitational mass; $\mathrm{x} \sim$ measurement of the fourth dimension of the gravity quality unit;

\subsection{The Intrinsic Property of the Gravitational Mass}

The gravity mass is placed into the three-dimensional space. According to the basic agreement (3), the gravity mass is acted on. The expression is as follows:

$$
\mathrm{M} \cdot \mathrm{C}=\frac{4}{3} \pi \cdot \mathrm{C} \cdot \mathrm{a}_{0}{ }^{3} \cdot \mathrm{b}_{0}{ }^{3} \cdot \mathrm{C}=\frac{4}{3} \pi \cdot \mathrm{F} \cdot \mathrm{x} \cdot \mathrm{a}_{0}{ }^{3} \cdot \mathrm{b}_{0}{ }^{3} \cdot \mathrm{F}_{1} \cdot|\mathrm{r}|
$$

In the form: $\mathrm{F} \sim$ the force of $3 \mathrm{~d}$ space; $|\mathrm{r}| \sim$ measurement of three-dimensional space in the direction of $\mathrm{F}$;

Definition: $\mathrm{M} \cdot \mathrm{C}$ is momentum $\mathrm{P}$ in the units of $\mathrm{m}^{5} / \mathrm{s}^{2}$ or $\mathrm{Kg} \cdot \mathrm{M} / \mathrm{s}$, which can be concluded as:

Conclusion 1 In $3 \mathrm{~d}$ space, the gravitational mass has intrinsic property of momentum.

When there is only one gravitational mass unit in $3 \mathrm{~d}$ space, the expression of momentum can only be itself. Therefore, the gravitational mass unit has spin angular momentum, which can be concluded as follows:

Conclusion 2 In $3 \mathrm{~d}$ space, the gravitational mass has intrinsic property of angular momentum.

Discussion:

(1) From equation 2, we can see that as long as the gravitational mass is put into three-dimensional space, the gravitational mass must have momentum. Therefore, in our world, momentum is the intrinsic property of the gravitational mass.

(2) On the right hand side of equation 2, there are two spatial and temporal combinations equal to $\mathrm{C}:(\mathrm{F} \cdot \mathrm{x})$ and $\left(\mathrm{F}_{1} \cdot|\mathrm{r}|\right),(\mathrm{F} \cdot \mathrm{x})$ is a combination of space-time constructed by the gravitational mass, and its action direction points to the fourth dimension. When the three-dimensional space is determined, for $(F \cdot x)$, the acting direction is perpendicular to the acting direction of three-dimensional space. $\left(\mathrm{F}_{1} \cdot|\mathrm{r}|\right)$ is the action direction of three-dimensional space, so $(\mathrm{F} \cdot \mathrm{x})$ and $\left(\mathrm{F}_{1} \cdot|\mathrm{r}|\right)$ are perpendicular to each other and do not have direct acting.

(3) In equation 2 , there is a change relation between $\left(F_{1}\right.$. $|r|)$ and $a_{0}$, because they are all three-dimensional quantities. $(F \cdot x)$ has a change relation with $b_{0}$, because $b_{0}$ represents the number of space cycles of four-dimensional gravitational mass and is also the density coefficient of gravitational mass.

(4) It can be judged from the two vertical interactions of the four-dimensional gravitational mass that the momentum of the four-dimensional gravitational mass has the characteristic of rotation. In other words, the $4 \mathrm{~d}$ gravitational mass represents the rotational angular momentum in $3 \mathrm{~d}$ space, so the rotational angular momentum is the intrinsic property of the $4 \mathrm{~d}$ gravitational mass.

(5) In equation 2 , neither $a_{0}$ nor $b_{0}$ is continuous. Therefore, the rotation angular momentum of the four-dimensional gravitational mass is discontinuous. In particular, $\mathrm{b}_{0}$ can only take the natural number positive integer.

\subsection{The Interaction of Gravitational Mass}

Suppose, put two gravitational masses of mass $\mathrm{M}_{1}$ and $\mathrm{M}_{2}$ into three-dimensional space, the distance between them is $\mathrm{r}$, in three-dimensional space, two gravitational masses have spin angular momentum, and the expression is:

$$
\begin{gathered}
P_{1}=M_{1} \cdot C=\frac{4}{3} \pi \cdot(F \cdot x) \cdot a_{1}{ }^{3} \cdot b_{1}{ }^{3} \cdot\left(F_{1} \cdot|r|\right) \\
P_{2}=M_{2} \cdot C=\frac{4}{3} \pi \cdot(F \cdot x) \cdot a_{2}{ }^{3} \cdot b_{2}{ }^{3} \cdot\left(F_{1} \cdot|r|\right.
\end{gathered}
$$

When there is only one gravitational mass, the gravitational mass spins in its fourth dimensional direction (the direction of the center of mass), and the gravitational mass has only spin angular momentum, When there are two gravitational masses, the momentum of the gravitational mass is broken down into two rotational momenta, one is its own center of mass, the other is the center of mass of the gravitational mass, Therefore, when two gravitational masses are placed in three-dimensional space, the gravitational masses will produce two states of mutual rotation and spin, that is, the gravitational masses will have spin angular momentum and rotational angular momentum, it can be concluded that:

Conclusion 3. In $3 \mathrm{~d}$ space, spin angular momentum and rotational angular momentum are intrinsic properties of gravity mass.

When multiple gravitational mass unit into the three dimensional space, interaction will happen, the spin angular momentum and rotation angular momentum will redistribute, This is a multi-body problem with a centroid system, and theoretical physics can be described in a good way, and the author is going to be discussing the microcosmic problems of the nucleus.

\subsection{The Space-Time Effect of Gravitational Mass}

Put the gravitational mass in three dimensions, according to 
the basic agreement(3), the gravitational mass has momentum, and it's got the change in velocity $\mathrm{C}$, which exists in every direction in three dimensions, It is assumed that the radius of gravity mass when it enters three-dimensional space is $r_{1}$, after entering three-dimensional space, the spatial and temporal distribution is redistributed according to the one-dimensional mass space-time transformation formula[6]:

$$
\left\{\begin{array}{l}
\frac{\mathrm{r}_{1}}{r_{2}}=1 /\left(1+\frac{1}{\frac{\mathrm{V}}{2 \mathrm{C}}+\sqrt{\frac{\mathrm{V}^{2}}{4 \mathrm{C}^{2}}+\frac{\mathrm{V}}{\mathrm{C}}}}\right) \\
\frac{\mathrm{F}_{1}}{\mathrm{~F}_{2}}=1 /\left(1+\frac{1}{\frac{\mathrm{V}}{2 \mathrm{C}}-\sqrt{\frac{\mathrm{V}^{2}}{4 \mathrm{C}^{2}}+\frac{\mathrm{V}}{\mathrm{C}}}}\right)
\end{array}\right.
$$

In the form: $r_{1}, F_{1}$ are the increment of space and the increment of force, $r_{2}$ and $F_{2}$ are the quantity of space and time after the redistribution, Since the increment of velocity obtained by the four-dimensional gravitational mass is positive, $F_{1}$ is positive and $r_{1}$ is negative. In the formula: $\mathrm{r}_{2}=\mathrm{r}-\mathrm{r}_{1}, \mathrm{~F}_{2}=\mathrm{F}-\mathrm{F}_{1}, \mathrm{~V}=\mathrm{C}$ can be substituted into the above formula:

$$
\mathrm{r}_{2} / \mathrm{r}=0.618033989, \quad \mathrm{~F}_{2} / \mathrm{F}=-1.618033989
$$

As can be seen from the above calculation, when the gravity mass enters the three-dimensional space, it will act, and the spatial scale of the gravity center of gravity shrinks and the force increases.

Due to the spatial contraction of the gravitational mass, the three-dimensional space is curved, thus producing the spatial effect of the gravitational mass.

Gravitational mass is four dimensional mass, Due to the action of pointing to the fourth dimension, the gravitational mass forms a spatial sphere with radius $r$, and the density coefficient of the spatial sphere is $b_{0}$, therefore, the surface space of gravity mass has curvature radius $r$ curvature, when the gravitational mass ACTS on the external three-dimensional space, the three-dimensional space will be curved and have curvature due to the effect of the basic agreement(3). Therefore, it can be concluded that:

Conclusion 4 spatial curvature is one of the representations of gravity mass.

We know that the curvature is inversely proportional to the radius of curvature. In the space of gravity mass itself, the curvature of space is determined by the density coefficient of the spatial sphere. In the outer three dimensional space of the gravitational mass, the curvature of space is determined by the reduced distance from the gravitational mass and the weight coefficient of the density. From equation 2, we can get:

$$
\frac{M}{\frac{4}{3} \pi a_{0}{ }^{3}}=\frac{F \cdot x \cdot b_{0}{ }^{3} \cdot F_{1} \cdot|r|}{C}=\rho
$$

In the form: on the left is the density of the gravitational mass, and on the right is a constant for the confirmed gravitational mass. When the mass remains constant, the density is inversely proportional to $\mathrm{a}_{0}{ }^{3}$, thus:

$$
\sqrt[3]{\frac{\rho}{\rho^{\prime}}}=\frac{a_{0}^{\prime}}{a_{0}} \rightarrow a_{0}^{\prime}=\sqrt[3]{\frac{\rho}{\rho^{\prime}}} a_{0} \rightarrow a_{0}=\sqrt[3]{\frac{\rho^{\prime}}{\rho}} a_{0}^{\prime}
$$

Define the equivalent radius of an object in $3 \mathrm{~d}$ space:

$$
\mathrm{R}=\sqrt[3]{\frac{\rho}{\rho_{0}}} R_{0}
$$

In the form: $\mathrm{R} \sim$ equivalent radius of the object, $\mathrm{R}_{0} \sim$ radius of the objects in three-dimensional space, $\sqrt[3]{\frac{\rho}{\rho_{0}}} \sim$ the object space conversion coefficient;

Similarly, we can define the equivalent forces of objects in 3d space:

$$
F=\sqrt{\frac{\rho}{\rho_{0}}} F_{0}
$$

Where: $\mathrm{F} \sim$ the equivalent forces of the object, $\mathrm{F}_{0} \sim$ object force in three dimensional space, $\sqrt{\frac{\rho}{\rho_{0}}} \sim$ the force equivalent coefficient of the object.

Equation 6 represents the radius of a four-dimensional gravitational mass unit equal to the object in a three-dimensional space with a gravitational mass.

Equation 7 represents the force of an object in a three-dimensional space with a gravitational mass equivalent to a four-dimensional gravitational mass unit of the same mass.

The spatial curvature of the surface of the gravity mass unit is:

$$
K_{0}=\frac{1}{a_{0}}
$$

At a certain point in the three-dimensional space outer of gravitational mass, the spatial curvature due to the spatial contraction of the gravitational mass unit is:

$$
K=\frac{1}{a_{0}+r}
$$

The spatial curvature caused by gravity is cumulative. When the gravitational mass is an object in three-dimensional space, the spatial curvature of any point outside it is:

$$
K=\sqrt[3]{\frac{\rho}{\rho_{0}}} \cdot \frac{1}{r} \cdot \int_{r}^{0} \frac{1}{R+r} d r
$$

Equation 8 is the space curvature equation of gravity mass; In the formula: $\mathrm{K} \sim \mathrm{a}$ bit of curvature in three-dimensional space, $\mathrm{R} \sim$ the equivalent radius of three-dimensional space objects, $\mathrm{r} \sim$ a bit of three-dimensional space to the distance of the object, $\rho \sim$ the density of the object, $\rho_{0} \sim$ the density of the gravity mass unit;

Equation 8 shows the effect of objects with gravitational mass on three-dimensional space, the effect is to make the curvature in the three-dimensional space of the object, and the 
size of the curvature is determined by equation 8 .

The average curvature of any two points in space can be calculated by the following formula:

$$
\bar{K}=\frac{K_{1}+K_{2}}{2}
$$

The calculation according to equation 5 shows that the surface of the gravity mass unit produces the change of force, as the internal force of gravity mass increases, the external force of gravity mass decreases, the force flows towards the gravitational mass and the center of mass. In the three-dimensional space outside the gravitational mass unit, the law of force reduction conforms to the inverse relation of distance, so the formation type;

$$
\frac{\Delta F}{F}=\frac{1}{1+r^{\prime}}
$$

When the gravitational mass is a three-dimensional object, the following formula is true:

$$
\frac{\Delta F}{F}=\sqrt{\frac{\rho}{\rho_{0}}} \cdot \frac{1}{1+r^{\prime}}
$$

Where, $\frac{\Delta F}{F}$ is the rate of change of force in the external three-dimensional space of gravitational mass, $\sqrt{\frac{\rho}{\rho_{0}}}$ is the equivalent coefficient of gravitational mass spatial force, and $r^{\prime}$ is the distance from a point in three-dimensional space to the equivalent radius of the object. When the radius of the object is $\mathrm{R}_{0}$, the equivalent radius is $\mathrm{R}$, and the shortest distance from the space to the object is $\mathrm{r}, r^{\prime}=R_{0}-R+r$, the above equation is written as:

$$
\frac{\Delta F}{F}=\sqrt{\frac{\rho}{\rho_{0}}} \cdot \frac{1}{1+R_{0}-R+r}
$$

According to the relation between force and time in the Matter Spatial Theory, the following formula can be obtained:

$$
\frac{-\Delta t}{t-\Delta t}=\sqrt{\frac{\rho}{\rho_{0}}} \cdot \frac{1}{1+R_{0}-R+r}
$$

The cumulative change rate of any two point forces in space is:

$$
\sum \frac{\Delta F}{F}=\sqrt{\frac{\rho}{\rho_{0}}} \cdot \int_{r_{1}}^{r_{2}} \frac{1}{1+R_{0}-R+r} d r
$$

\subsection{The Gravitational Equation of the Gravitational Mass}

Multiply both sides of equation 2 by force F' and we get:

$$
M \cdot C \cdot F^{\prime}=\frac{4}{3} \pi \cdot C^{2} \cdot a_{0}{ }^{3} \cdot b_{0}{ }^{3} \cdot F^{\prime}
$$

On the left side of equation 13 is the "acting force" of the gravitational mass in units of $\mathrm{m}^{5} / \mathrm{s}^{3}=\mathrm{Kg} \cdot \mathrm{m} / \mathrm{s}^{2}$, this unit is consistent with the mechanical unit "Newton", and on the right is the force - space structure of the gravitational mass.

In the three-dimensional space, two gravitational masses
$\mathrm{M}_{1}$ and $\mathrm{M}_{2}$ are introduced, and the two gravitational masses with "acting force" interact with each other, which is expressed as follows:

$$
M_{1} \cdot C \cdot M_{2} \cdot C \cdot F^{\prime 2}=\frac{4}{3} \pi \cdot C^{2} \cdot a_{1}{ }^{3} \cdot b_{1}{ }^{3} \cdot \frac{4}{3} \pi \cdot C^{2} \cdot a_{2}{ }^{3} \cdot b_{2}{ }^{3} \cdot F^{\prime 2}
$$

Finishing:

$$
M_{1} \cdot M_{2} \cdot F^{\prime 2}=\frac{4}{3} \pi \cdot C \cdot{a_{1}}^{3} \cdot b_{1}{ }^{3} \cdot \frac{4}{3} \pi \cdot C \cdot a_{2}{ }^{3} \cdot b_{2}{ }^{3} \cdot F^{2}
$$

Since the gravitational mass $\mathrm{M}_{1}$ and $\mathrm{M}_{2}$ cannot be considered as particles in the micro domain, the action direction of the gravitational mass is only in the fourth dimension, and the coefficient is $1 / 4$, the action cannot be calculated repeatedly, $\pi \cdot C$ convert $\sqrt{\pi \cdot C}$, therefore, the above expression can be rewritten as:

$$
\begin{aligned}
M_{1} \cdot M_{2} \cdot \frac{1}{16 \pi C} \cdot F^{\prime 2} & =\frac{1}{4} \cdot \frac{4}{3} \sqrt{\pi \cdot C} \cdot a_{1}{ }^{3} \cdot b_{1}{ }^{3} \cdot \frac{1}{4} \cdot \frac{4}{3} \sqrt{\pi \cdot C} \cdot a_{2}{ }^{3} \cdot b_{2}{ }^{3} \\
\cdot & F^{\prime 2}
\end{aligned}
$$

Divide both sides by the square of the distance and we get:

$$
\frac{M_{1} \cdot M_{2}}{r^{2}} \cdot \frac{F^{\prime 2}}{16 \pi C}=\frac{F^{\prime 2}}{9 r^{2}} \pi \cdot C \cdot a_{1}{ }^{3} \cdot b_{1}{ }^{3} \cdot a_{2}{ }^{3} \cdot b_{2}{ }^{3}
$$

We define the interaction force generated by the gravitational mass under the force of $F^{\prime}=1 / 1 \mathrm{~s}$ is gravitation, and the unit is Newton $\mathrm{N}$, or $\mathrm{m}^{5} / \mathrm{s}^{3}$ or $\mathrm{Kg} \cdot \mathrm{m} / \mathrm{s}^{2}$, it can be written as follows:

$$
A_{F}=\frac{M_{1} \cdot M_{2}}{r^{2}} \cdot \frac{1}{16 \pi C}
$$

In the form of: $A_{F} \sim$ gravity, $\mathrm{M} \sim$ gravity mass, $\mathrm{r} \sim$ gravity mass distance between;

Make: $G=\frac{1}{16 \pi C}=6.6360468 \cdot 10^{-11}$ as the gravitational constant, It can be extended to the macro field.

Therefore, it can be concluded that:

Conclusion 5 Gravity is the representation of gravity mass when the force is $1 / 1 \mathrm{~s}$.

Discussion:

(1) Formula 16 can be interpreted as two gravitational mass extending space " $r$ " the product of the mass flow density.

(2) "Acting force" AF is a relative physical quantity, which cannot strictly correspond to the intrinsic property of gravitational mass. When we specify that the force of the system is 1, mass flow can be expressed as gravitational mass $\mathrm{M}$, the distribution of mass flow along the $r$ direction is the mass flow density, and the force is the property of the product of mass flow density in $3 \mathrm{~d}$ space.

(3) The gravitational constant $G=\frac{1}{16 \pi C} \quad$ in the macroscopic domain is not a mathematical constant without physical significance, it represents the actual range of the interaction between the gravitational masses and the concrete manifestation of the effect of the gravitational masses. 


\section{Experimental Verification}

\subsection{Light Ray Refraction in Gravitational Field}

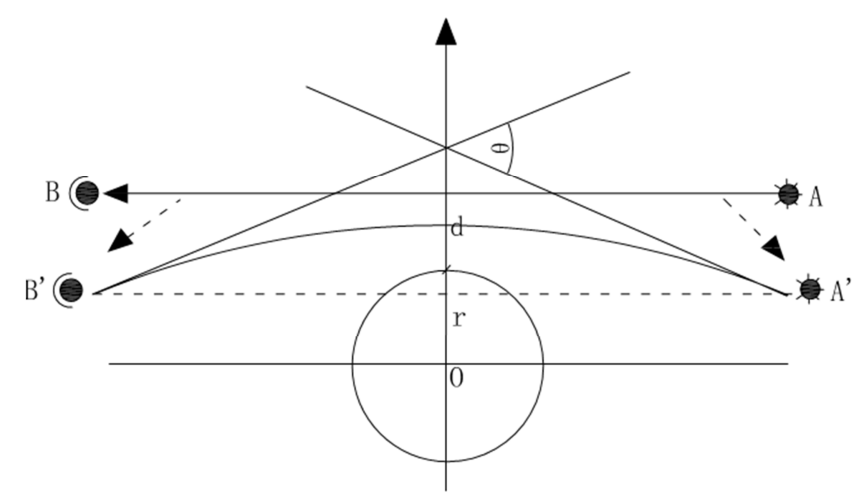

Figure 1. Curvature of space due to gravitational mass.

As shown in figure 1, the observer is at point B, the light emitted from point $A$ is observed, and the gravitational mass is at point $\mathrm{O}$, when there is no gravitational mass, line $\mathrm{AB}$ is the direction of observation, after placing the gravitational mass with radius $r$ at point $\mathrm{O}$, Because of the constraint of the principle of constant speed of light, the radius of the gravitational mass produce $\Delta \mathrm{R}$ contraction, the curvature of space at the edge of gravity mass, with definite curvature. As the light passes, the light path deflects in the direction shown by the dashed arrow, the actual light path is the arc shown in $\mathrm{A}^{\prime}$ and $\mathrm{B}^{\prime}$. The tangent line is made at points $\mathrm{A}$ 'and $\mathrm{B}$ ', where the Angle $\theta$ is the deflection Angle of the observer, At the same time, the Angle $\theta$ is also the tangent Angle to calculate the curvature of A 'and B' section curves, we get:

$$
K=\frac{\theta}{\widetilde{A^{\prime} B^{\prime}}}
$$

The average curvature of A'and B' curves can be calculated according to equations 8 and 9:

Equation 8: $K=\sqrt[3]{\frac{\rho}{\rho_{0}}} \cdot \frac{1}{r} \cdot \int_{r}^{0} \frac{1}{R+r} d r$ Equation 9: $\bar{K}=$ $\frac{K_{1}+K_{2}}{2}$, After integration, we can get:

$$
\begin{array}{r}
\bar{K}=\frac{1}{r} \cdot \sqrt[3]{\frac{\rho}{\rho_{0}}}[\ln (R+r)-\ln (R)\rceil \\
\sum\left(\frac{-\Delta t}{t-\Delta t}\right)_{+}=\sqrt{\frac{\rho}{\rho_{0}}} \cdot\left[\ln \left(1+R_{0}-R+r\right)-\ln (1+R)\right\rceil \\
\sum\left(\frac{-\Delta t}{t-\Delta t}\right)_{-}=\sqrt{\frac{\rho}{\rho_{0}}} \cdot\left[\ln (1+r)-\ln \left(1+r-R+R_{0}\right)\right\rceil
\end{array}
$$

The effect of gravity mass field on time is a cumulative result, therefore, the integral of the above equation is required, the integral range leaving the gravitational field is: 0 ? $\left(R_{0}-\right.$ $R+r)$, the integral range into the gravitational field is: $\left(r+R_{0}-R\right) ? r$, if the departure direction is positive and the entry direction is negative, then:

The gravitational mass equivalent radius of action: $\mathrm{r}^{\prime}=1.048602719 \cdot 10^{-15} \mathrm{~m}$, Mass of proton : $1.6726213678 \cdot 10^{-27} \mathrm{Kg}$, Calculate the density as: $3.46318875 \cdot 10^{17} \mathrm{Kg} / \mathrm{m}^{3}$, Density of sun: $\rho=1409 \mathrm{Kg} / \mathrm{m}^{3}$, Radius of sun: $\mathrm{R}_{0}=6.955 \cdot 10^{8} \mathrm{~m}$, The distance from the sun to the earth: $\mathrm{r}=1.496 \cdot 10^{11} \mathrm{~m}$, Acquire by calculation: $\bar{K}=$ $1.75170128 \cdot 10^{-15} 1 / \mathrm{m}$

According to the curvature formula, the arc length is approximately replaced by $2 \mathrm{R}$, and thus:

The calculation of general relativity is $\theta=1$ ".75, actual observation is $\theta=1 " .61 \sim 1 " .98$. [7, 8]

These results are consistent with the results of general relativity calculation and experimental observation.

\subsection{Gravitational Redshift}

According to the properties of the gravitational mass, the force is increasing in the direction of the center of mass on its surface, corresponding to the decrease in time, in the direction way from the center of mass, the force is decreasing, sponding to the increase in time, therefore, in the

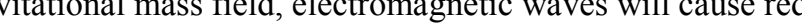
gravitational mass shift red and entering the gravitational mass field shift blue.

Objects in nature need to be reduced to a standard gravitational mass density for calculation. According to the properties of the gravitational field, the influence result is inversely proportional to the distance, and equation 12 can be used to calculate the time influence coefficient:

$$
\sum \frac{-\Delta t}{t-\Delta t}=\sqrt{\frac{\rho}{\rho_{0}}} \cdot \int_{r_{1}}^{r_{2}} \frac{1}{1+R+r} d r
$$

The electromagnetic waves coming from the sun are affected by the gravity of the earth and the sun. They have the opposite effect. Therefore, the time effect of observing the spectrum from the sun on the earth is:

$$
\delta t=\delta t_{\text {earth }}+\delta t_{\text {sun }}
$$

In the mass field, the time effect through the distance of $\mathrm{R}$ is:

$$
\delta=\sum\left(\frac{-\Delta t}{t-\Delta t}\right)_{+}+\sum\left(\frac{-\Delta t}{t-\Delta t}\right)_{-}
$$


According to the relationship between frequency and time:

$$
\frac{\Delta v}{v_{0}}=\sum \frac{-\Delta t}{t-\Delta t} \approx-\sum \frac{\Delta t}{t}
$$

Gravity mass density $3.46318875 \cdot 10^{17} \mathrm{Kg} / \mathrm{m}^{3}$ was taken as the standard mass density, density of sun: $\rho=1409 \mathrm{Kg} / \mathrm{m}^{3}$, density of earth: $\rho_{2}=5507.85 \mathrm{Kg} / \mathrm{m}^{3}$, radius of sun: $R_{0}=6.955 \cdot 10^{8} \mathrm{~m}$, equivalent radius of sun: $\mathrm{R}=1.1102298 \cdot 10^{4} \mathrm{~m}$, radius of earth: $\mathrm{R}_{0}=6371393 \mathrm{~m}$, equivalent radius of earth: $\mathrm{R}=160.22725 \mathrm{~m}$, the distance from the sun to the earth: $\mathrm{r}=1.496 \cdot 10^{11} \mathrm{~m}$, acquire by calculation:

$$
\begin{aligned}
& \delta=\delta_{\text {earth }}+\delta_{\text {sun }} \\
&=+5.370758 \cdot 10^{-12}-1.04729689 \\
& \cdot 10^{-6} \approx-1.04729689 \cdot 10^{-6}
\end{aligned}
$$

The spectrum of the sun observed on earth is redshift, and the effect of the earth's gravitational field on the result is small and can be ignored.

The result of general relativity is $2.12 \cdot 10^{-6}$.[9]

On the surface of the earth, electromagnetic wave shooting from the ground to $\mathrm{H}$ height will produce redshift, and its effect is the difference between the effect of $\mathrm{H}$ height and the effect of the ground. The formula can be calculated as follows:

$$
\sum \frac{-\Delta \mathrm{t}}{\mathrm{t}-\Delta \mathrm{t}_{+}}=\sqrt{\frac{\rho}{\rho_{0}}} \cdot\left[\ln \left(1+\left(\mathrm{R}_{0}-\mathrm{R}\right)\right)-\ln \left(1+\mathrm{H}+\left(\mathrm{R}_{0}-\mathrm{R}\right)\right)\right]
$$

When $\mathrm{H}$ is $22.5 \mathrm{~m}$ :

$$
\frac{\Delta \mathrm{v}}{\mathrm{v}_{0}}=-4.45335997 \cdot 10^{-13}
$$

When $\mathrm{H}$ is $12.5 \mathrm{~m}$ :

$$
\frac{\Delta \mathrm{v}}{\mathrm{v}_{0}}=-2.47422401 \cdot 10^{-13}
$$

The ratio of 12.5 meters to 22.5 meters is 0.55556

The result of general relativity $[10,11]$ is $2.46 \cdot 10^{-15}$ and $1.36 \cdot 10^{-15}$, the ratio of 12.5 meters to 22.5 meters is also 0.55556 .

There is a large gap between our calculation results and those of general relativity, which needs to be analyzed.

When emitted from $\mathrm{H}$ height to the ground, the electromagnetic wave will produce blue shift, which is the same as above.

\subsection{Observation of Echo delay of Electromagnetic Wave}

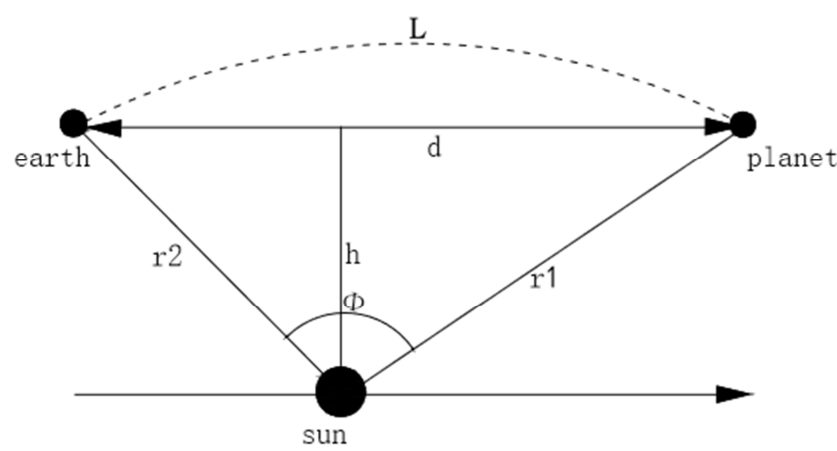

Figure 2. Delay in echo of electromagnetic waves emitted by the earth to the planet.

Electromagnetic waves emitted by the earth, when there is no solar gravity, are flat in space. Therefore, electromagnetic waves travel in a straight line, and the time of echo receiving is:

$$
\mathrm{t}=\frac{2 \mathrm{~d}}{\mathrm{C}}
$$

Due to the gravitational effect of the sun, the receiving time of echo is:

$$
\mathrm{t}^{\prime}=\frac{2 \mathrm{~L}}{\mathrm{C}}
$$

The average curvature of two points can be calculated according to equations 8 and 9:

$$
\overline{\mathrm{K}}=\frac{\overline{\mathrm{K}}_{\text {earth }}+\overline{\mathrm{K}}_{\text {mercury }}}{2}
$$

The radius of curvature is:

$$
\mathrm{R}=\frac{1}{\overline{\mathrm{K}}}
$$

Given the chord length and radius, the arc length L can be obtained as follows:

$$
\begin{gathered}
\mathrm{L}=\frac{2}{\overline{\mathrm{K}}} \cdot \sin ^{-1}\left(\frac{\overline{\mathrm{K}} \cdot \mathrm{d}}{2}\right) \\
\mathrm{t}^{\prime}=\frac{2 \mathrm{~L}}{\mathrm{C}}=\frac{4}{\mathrm{C} \cdot \overline{\mathrm{K}}} \cdot \sin ^{-1}\left(\frac{\overline{\mathrm{K}} \cdot \mathrm{d}}{2}\right) \\
\Delta \mathrm{t}=\frac{2 \mathrm{~L}}{\mathrm{C}}-\frac{2 \mathrm{~d}}{\mathrm{C}}=\frac{4}{\mathrm{C} \cdot \overline{\mathrm{K}}} \cdot \sin ^{-1}\left(\frac{\overline{\mathrm{K}} \cdot \mathrm{d}}{2}\right)-\frac{2 \mathrm{~d}}{\mathrm{C}}
\end{gathered}
$$

By observing the delay of mercury's electromagnetic echo, we can obtain:

The earth's distance from the sun is $r_{2}=1.496 \cdot 10^{11} \mathrm{~m}$, mercury's distance from the sun is $\mathrm{r} 1=5.790905 \cdot 10^{10} \mathrm{~m}$ (mean value), mercury's distance from the earth is $\mathrm{d}=2 \cdot 10^{11} \mathrm{~m}$, the sun's radius is $6.955 \cdot 10^{8} \mathrm{~m}$, the equivalent radius of sun is $1.1102298 \cdot 10^{4} \mathrm{~m}$, by substituting equations 8 and 9 , it can be obtained: $\mathrm{K}=3.007677378 \cdot 10^{-15}, \mathrm{~L}=2.00000003019 \cdot 10^{11} \mathrm{~m}$, the calculation time delay is:

$$
\Delta \mathrm{t}=\frac{2(\mathrm{~L}-\mathrm{d})}{\mathrm{C}}=2.01406 \cdot 10^{-5} \mathrm{~s}
$$

The result of general relativity is $2.4 \cdot 10^{-4} \mathrm{~s}$. $[12,13]$

There is a large gap between our calculation results and those of general relativity, which needs to be analyzed. 


\section{Discussion}

Based on the fundamental principles of the Matter Spatial Theory, we discuss the intrinsic property of gravity mass, in the condition of the invariable principle of the speed of the light, we explain the generation and evolution of gravity from the basic properties of the space-time structure. We found that Spin angular momentum and rotational angular momentum are intrinsic properties of gravitational mass, and space curvature and gravitational force are representations of gravitational mass. Mass flow density is the source of gravitational "acting force".

We derived the equation of gravitation from the four dimensional mass expression in the Matter Spatial Theory. In particular, we get the mathematical expression for the gravitational constant $\mathrm{G}=1 / 16 \pi \mathrm{C}$, it provides a possible direction for the physical source of the gravitational constant.

Based on the theory of the Matter Spatial Theory, we get the law of space-time change in the gravitational field, and derive the equation of space curvature and the equation of time effect.

We used the calculation results of general relativity and the experimental results of verification ${ }^{[10]}$ to test our equations. The solar starlight deflection experiment is in good agreement with our theoretical calculation (the relative error less than $3 \%$ ).

In the experiments of gravitational redshift and radar echo delay, the results of our theoretical calculation differ greatly from those of general relativity, which may be due to the calculation parameters of standard gravity density.

In the red shift experiment of electromagnetic wave emission on the ground, our calculation results are consistent with the linear relation of the experimental results, the red shift ratio of electromagnetic wave at the height of $12.5 \mathrm{~m}$ and $22.5 \mathrm{~m}$ is consistent with the experimental results, which are both 0.555556 , it is suggested that there may still be a coefficient factor missing in our calculation formula, which provides a clue for our subsequent research.

\section{Conclusions and Inferences}

Through the research in this paper, we have determined that the intrinsic property of gravitational mass is spin angular momentum and spin angular momentum. This result is the property of all objects with gravitational mass at present, and no exceptional phenomenon has been observed.

Through the research in this paper, we believe that the representation of gravitational mass is a "gravitational phenomenon", while the essence of gravitational phenomenon is the "spatial curvature of gravitational mass". The formation of spatial curvature is due to the fact that the gravitational mass is a four-dimensional mass, and the four-dimensional mass exists in three-dimensional space to point to the space contraction of the fourth dimension. The curvature of space around the gravitational mass can be indirectly proved by the deflection of starlight around the sun.

Through the study of this article, we can sure, gravitational constant is not a mathematical constant no physical meaning, gravitational constant is gravitational mass between scope and effect way, by the formula $\mathrm{F}^{2} / 16 \pi \mathrm{C}$ as you can see, the gravitational constant is related to time rule, when the time rule for 1 , gravitational constant is $1 / 16 \pi C$, studies have shown that gravitational constant is related to time[14].

Through the study in this paper, we can infer that space in the universe has curvature. When the density of celestial bodies does not change, the curvature of space remains unchanged, and we cannot observe the gravity phenomenon. It is only when the density of an object changes significantly that the curvature of space around it changes, and we should be able to observe the changes in the curvature of space. Therefore, we can observe the decrease of space curvature caused by supernova explosion and the increase of space curvature caused by neutron star formation, and these changes will produce the light effect of surrounding space.

It can be inferred from the research in this paper that graviton is not required for the transfer of gravity, Gravity is transmitted through changes in spatial curvature. The observation of gravitational wave can only prove the change of space curvature, but not the existence of gravitons.

\section{References}

[1] Carmeli, M.: Classical Field-General Relativity and Gauge Theory(1982).

[2] Adler, R., Bazin, M and Schiffer, M.: Introduction to General Relativity, Zndedi (1975).

[3] arXiv:0909.3583v4 Virgo Collaboration: B. P. Abbott, R. Abbott, F. Acernese, Etc. Searches for gravitational waves from known pulsars with S5 LIGO data, LIGO Scientific Collaboration, Etc.

[4] Lawrence M. Krauss, Frank Wilczek. Using Cosmology to establish the Quantization of Gravity Phys. Rev. D 89,047501(2014).

[5] Haitao Gao, The Matter Spatial Theory [M] Xinhua Book Co. Ltd. June 2018 Hong Kong.

[6] Haitao Gao, The space-time transformation in the velocity field, Beifang University of Nationalities.

[7] F. W. Dyson, A. S. Eddington and C. Davidson, A Determination of the Deflection of Light by the Sun's Gravitational Field, from Observations Made at the Total Eclipse of May 29, 1919 Phil. Trans. R. Soc. Lond. A 1920220.

[8] Brault J. The gravitational redshift in the solar spectrum. Bull Amer Phys S OC, 1963, 8:28.

[9] J E, Roddier F. Precise observation of the profile of the fraunhofer strontium resonance line evidence for the gravitational red shift on the sun. Phys Rev Lett, 1961, 7:437.

[10] Granshow TE, et al. Measurement of the gravitational red shift using the Mossbauer effect in Fe57. Phys Rev Lett, 1960, 4(4):163.

[11] Pound R V, Snider J L. Effect of gravity on gamma radiation. Phys Rev B, 1965, 140:788. 
[12] I. I. Shapiro, et al, Phys. Bev. Lett. 20, 1205(1968).

[13] I. I. Shapiro, et al, Phys. Bev. Lett. 26, 1132(1971).
[14] R. W. Hellings, in Proc. 10 the International Conference on General Relativity and Gravitation, ed. B. Bertotti et. al. (Consiglio Nazionale Delle Ricerche-Rome, 1983). 